\title{
La «mort de la belle jeunesse» ou le suicide juvénile à Genève au $\mathrm{XVIII}^{\mathrm{e}}$ siècle
}

\author{
par Michel Porret
}

\section{Résumé}

Terme solitaire de chagrins familiaux, amoureux ou de pathologies douloureuses, le suicide juvénile horrifie les communautés de l'Ancien Régime promptes dès le début du XVIII e siècle à y discerner une "maladie de l'âme» particulière à une jeunesse qui conjugue vitalité corporelle et fragilité morale et dont le rôle est d'assurer les lendemains du genre humain. Grossissant le ressentiment social à l'encontre de la mort volontaire attribuée souvent depuis 1774 à l'exemple pernicieux de la "philosophie» ou du libre-arbitre de l'infortuné Werther, le suicide juvénile souligne les failles de la solidarité sociale dans la communauté resserrée de l'Ancien Régime.

\section{I}

Consacré aux dossiers pénaux du suicide juvénile, cet article veut examiner la perception morale et sociale du chemin suicidaire qu'empruntent une cinquantaine de désespérés qui se tuent volontairement à Genève entre 1750 et $1790^{1}$.

Dans la République protestante, les archives criminelles montrent dès le début du XVIII ${ }^{e}$ siècle une modernisation précoce de la pratique pénale, notamment avec la mise en place d'une médecine légale systématique lors de crimes de sang ou d'attentats aux mœurs, la disparition des supplices les plus archaïques (celui de la roue est infligé une ultime fois en 1728), l'abandon en 1738 de la question nécessaire à extirper l'aveu et le renforcement ininterrompu du pouvoir judiciaire du Procureur général qui détermine la légalité des peines selon une jurisprudence locale ou «universelle», les «circonstances» du crime et la doctrine imprimée. A cet «adoucissement» procédurier, salué par exemple en 1752 dans l'article Genève de l'Encyclopédie de Diderot et d'Alembert, s'ajoute le recul inexorable de la peine capitale jugée inefficace à véritablement corriger les «scélérats», alors que depuis les années 1760 les procureurs généraux, adversaires de l'infamie qui flétrit corps et 
âme mais ne corrige point, plaident pour un projet carcéral que les institutions genevoises ne permettent pas encore de réaliser ${ }^{2}$. Emportant un adoucissement des châtiments que permet une criminalité de faible envergure, cette modernisation du régime judiciaire de Genève, toujours soumis à l'arbitraire des juges jusqu'au Code pénal genevois de $1795^{3}$, se remarque aussi dans la nature de la répression du suicide perçu par maints magistrats et pasteurs depuis la fin des années 1730 comme une maladie de l'âme plutôt qu'un crime selon l'esprit de la loi.

«J'ai cherché en vain dans les Correspondances, les Mémoires, les romans, un récit montrant des gens du peuple face à face avec le suicide»,

déplore à l'aube de notre siècle le sociologue Bayet ${ }^{4}$. Jusqu'aux années 1950, l'histoire de la criminalité et de l'anomie sociale, se confinait à une statistique «explicative» ou à une analyse des doctrines savantes. Or l'histoire de la «perception morale» du suicide au sein des communautés de l'Ancien régime doit se doubler d'une patiente enquête à travers les archives judiciaires. En effet, depuis l'Ordonnance criminelle de 1670 qui pénalisait en France l'«homicide de soi-même», décriminalisé avec le premier Code pénal de 1791, le suicide est un délit qui emportait flétrissure post-mortem du cadavre, inhumation hors du cimetière et confiscation des biens personnels du suicidé ${ }^{5}$. Menées dans les archives criminelles, ces études confirment la leçon de l'anthropologie montrant la complexité historique du «suicide» dont le sens social varie à travers temps et civilisations ${ }^{6}$. Marquée par la tolérance de Montesquieu, Voltaire ou Helvétius, l'opinion lettrée de la seconde moitié du $\mathrm{XVIII}^{\mathrm{e}}$ siècle, toujours hostile au suicide, commençait cependant d'y voir le signe d'une «maladie» guérissable plutôt qu'un crime. Pourtant, les théologiens, jurisconsultes, philosophes et moralistes de tous bords fustigèrent jusqu'au terme du XVIII e siècle le suicide, ce «néologisme aberrant» apparu en $1732^{7}$, encore défini en 1771 par Le Dictionnaire de Trévoux comme le «système des lâches». Semblable peut-être à l'épilepsie entourée d'une «antique superstition», dont Tissot voulait effacer la «fausse honte qu'on y attache», le suicide se débarrassait ainsi lentement de son cortège de "préjugés populaires» ${ }^{8}$ toujours inspirés par l'idée du péché que défendit l'Eglise jusqu'à la fin de l'Ancien Régime. Décriminalisant le suicide en 1791, le Code pénal entérine finalement une réalité socialement accomplie et souligne, une fois de plus, l'abîme entre droit pénal et opinion publique ${ }^{9}$.

Alors que les conservateurs catholiques espèrent recriminaliser le suicide durant la Restauration ${ }^{10}$, au XIX ${ }^{\mathrm{e}}$ siècle, les aliénistes défendent bientôt la 
thèse de la démence: «Les maniaques se tuent», affirme Esquirol qui voit la mort volontaire comme le symptôme d'une «maladie mentale» altérant physionomie et physiologie humaines. Source d'atavisme familial, «acte consécutif du délire des passions ou de la folie», le suicide, l'un «des objets les plus importants de la médecine clinique», prouve la «monomanie [qui] peut conduire au meurtre de soi-même» ${ }^{11}$. Maladie d'esprits débiles chez les adultes, le suicide, "plus fréquent depuis l'âge de vingt ans jusqu'à trente» ${ }^{12}$, exprime au seuil de l'ère industrielle le désœuvrement juvénile face au «changement moral» produit par l'effondrement du modèle familial traditionnel. Avant l'école psychanalyste discutant de l'équilibre fragile entre «pulsion de vie» et "pulsion de mort» utilisé par Freud dès 1920 (Eros et destruction), la sociologie durkheimienne avait proposé un paradigme déterministe à la signification du suicide, manifestation de la pathologie sociale $^{13}$. Selon Durkheim, le suicide est altruiste lorsque le sujet se tue par dévouement collectif; au contraire le suicide anomique provient d'une représentation intime de l'idée de solitude produite par un affaiblissement des liens communautaires. Finalement, le suicide égoïste naît d'un culte exacerbé du moi (Narcisse), peut-être particulier à la jeunesse ravagée par les passions. Pour Durkheim, la fréquence de la morbidité suicidaire augmente d'une façon inverse aux possibilités d'intégration de la société religieuse, familiale ou politique. Les communautés protestantes, qui ont aboli le rite compensatoire de la confession et valorisent d'une manière exacerbée la réussite individuelle, induisent un univers de culpabilité particulièrement propice à la mort volontaire. Dans la République protestante, entre 1750 et la fin du XVIII ${ }^{e}$ siècle, les suicides juvéniles concluent souvent des conflits de jeunes adultes qui se détachent de la communauté, voire se marginalisent par chagrin, douleur ou exaltation.

\section{II}

A Genève, en 1779, un Auditeur (magistrat instructeur), fouillant parmi les papiers laissés par un adolescent qui s'est tiré une balle dans la tête, écrit sur le «verbal» de perquisition:

\footnotetext{
«Nous étant fait représenter le livre trouvé sur le dit jeune homme. Nous avons vu que c'etoit un livre traduit de l'allemand intitulé $L a$ Vie et les souffrances du jeune Werther. Livre qui contient l'histoire d'un jeune homme qui termine sa vie par un coup de pistolet, après avoir écrit de longues réflections sur la mort qu'il se prépare» ${ }^{14}$.
} 
Dernière lettre inspirée par la prière ou les formules testamentaires que laissent 36 individus âgés de moins de trente ans qui confient leur «âme à Dieu», livres ouverts à la page d'un poème particulier, estampes érotiques abandonnées, voire traité d'inspiration naturaliste sur le suicide: la mise en scène autour du suicide juvénile se multiplie à Genève depuis 1750 et permet de mesurer les rapports culturels nouveaux qui se développent dans le contexte d'une alphabétisation progressive autour de la lecture et des modèles littéraires ${ }^{15}$. Enoncées dans la «dernière lettre», les causes explicites du suicide mêlent réparation d'honneur, chagrin amoureux ou grande émotivité particulière à la «mélancolie, cette humeur noire qui conduit au tombeau». Multiple et individuelle, la causalité avouée du suicide exprime avant tout un «désir de ne pas mourir tout entier» ${ }^{16}$ tant est aiguë la volonté des désespérés de ne point sombrer dans l'oubli.

Pour les magistrats genevois alarmés par l'«épidémie de suicide» qu'aurait produit Goethe au moment de publier en 1774 Die Leiden des jungen Werther, texte qui souleva les prêtres et les autorités morales de l'Europe des Lumières contre le modèle pernicieux des «lectures philosophiques» ${ }^{17}$, la dernière lettre laissée pour la famille résume les raisons avouées du geste fatal, alors que les ouvrages épars sur le sol de la soupente solitaire apparaissent quelquefois comme la cause directe de la mort volontaire. Durant les deux dernières décennies de l'Ancien Régime, cette "philosophie de la mort» aurait contaminé la jeunesse européenne, instruite et urbaine, affectée d'une mélancolie inguérissable. Proche du mythe culturel, l'impact réel des «lectures philosophiques» sur la courbe des suicides n'a jamais été démontré. Au $\mathrm{XVIII}^{\mathrm{e}}$ siècle, l'inquiétude des prêtres, officiers de justice, autorités politiques et opinion publique, traduit une attention nouvelle à cette «impasse sociale» qu'est le suicide. Avec étonnement, au fil des fleuves ou dans les greniers des capitales européennes, on découvre la cohorte livide des suicidés, non point en raison d'une recrudescence subite des noyades volontaires ou des pendaisons provoquées par la lecture de Werther, mais plutôt parce que le désir de mort émerge de son ombre séculaire d'infamie pénale et d'opprobre social ${ }^{18}$.

Or les suicides commis par des adolescents ou des adultes jeunes nourrissent l'inquiétude des cliniciens du XIX ${ }^{\mathrm{e}}$ siècle, puis des sociologues, anthropologues et psychiatres contemporains attachés à cerner les racines du «mal de vivre» ${ }^{19}$. La mort volontaire de la «belle jeunesse» ne montre-t-elle pas la nature agressive des «représailles» affectives adressées à pères et mères avides de cet amour nécessaire au développement de l'individu? Hier ou 


\title{
T R A I T E D U
}

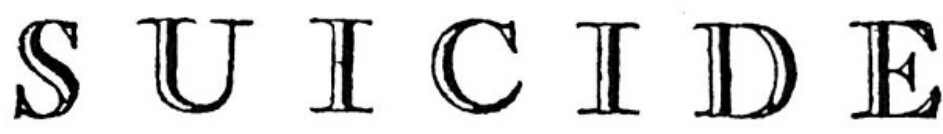

OU DU MEURTRE VOLONTAIRE , DE SOI - MEME.

$P_{A R}$ FEAN DUMAS.

\begin{abstract}
Savoir fouffrir la vie $\&$ voir venir la mort, C'eft le devoir du Sage, \& tel fera mon fort. Grefet Trag. d'Edouard III.
\end{abstract}

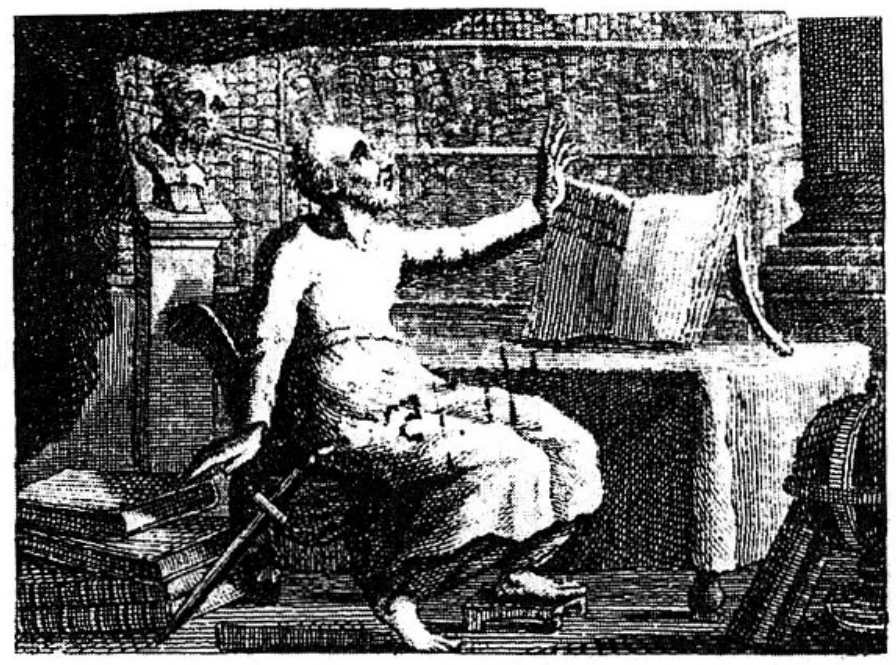

\section{A $A M S T E R D A M$, \\ C н E z D. J. C H A N G U I O N. $M D C C L X \times I I I$.}

Fortifier l'âme, apaiser les inquiétudes et attiser la foi : jusqu'à la fin de l'Ancien Régime telle est la leçon de multiples traités philosophiques ou moraux qui fustigent la mauvaise mort apportée par le suicide et dénoncée ici par le pasteur protestant Jean Dumas. 
aujourd'hui, le suicide juvénile altère l'image du bonheur familial et plonge la famille endeuillée dans le monde du remords et de la culpabilité.

L'auteur anonyme d'un article intitulé De la Vieillesse, publié en 1757 dans Le Conservateur ${ }^{20}$, exalte le suicide du vieillard dont le corps et l'esprit se dégradent. Inspiré aux consignes lucides de Sénèque, ce diagnostic stoïcien répond au radicalisme matérialiste de cette «philosophie de la Nature»

qui définissait la «vieillesse [comme] le tombeau de l'intelligence et de la sensibilité», pathologie léthargique où les «idées s'effacent, les sensations s'affaiblissent [où] on ne sent plus le présent, on perd la trace du passé, et on ne jette que des regards tremblans sur l'avenir» ${ }^{21}$.

Seule cette «éclipse de l'entendement» justifie le suicide, car, continue le Philosophe, la jeunesse nourrie par des «passions douces» ne peut oublier l'«horizon de la vie» fait d'un lendemain heureux ${ }^{22}$.

La jeunesse, qui «s'étend depuis environ 21 ans jusqu'à $35 »$, mêle vitalité corporelle et immaturité intellectuelle, puisque le corps, «après avoir acquis les dimensions qui lui conviennent, achève de se perfectionner acquérant toute la force et la solidité nécessaire à sa conservation» ${ }^{23}$, $n$ 'est que la mince enveloppe de la fragilité morale. Moment de plénitude corporelle, pourtant âge où les «passions excessives», ces «maladies de l'âme» selon le diagnostic rationnel des médecins et moralistes des Lumières ${ }^{24}$, peuvent amener à des comportements néfastes à la "préservation» de la vie, notamment lorsque la «fureur de vivre» débouche sur la mort. L'imaginaire médical, obsédé par la dispersion des énergies vitales qu'illustre la «manie» onanique détournant de la reproduction sexuelle au profit des abîmes de la volupté solitaire, ne perçoit-il pas quelquefois le suicide juvénile comme l'issue perverse qui menace les damnés de la masturbation ${ }^{25}$ ? Scandale singulier, pour le clinicien du $\mathrm{XIX}^{\mathrm{e}}$ siècle, que la mort de ce garçonnet de treize ans, livré à ses passions délétères, qui se pend en laissant ce billet d'adieu : «Je lègue mon âme $\grave{a}$ Rousseau et mon corps à la terre» ${ }^{26}$.

L’apologie du néo-stoïcien et le bilan sociologique alarmé devant la crue des suicides juvéniles amènent une interrogation nouvelle: comment la jeunesse peut-elle devenir le creuset moral où se détermine la fin brutale de l'existence? Existe-t-il un modèle particulier de la conduite suicidaire et du suicide juvénile propres à ce monde disparu qui précède l'ère industrielle, réputé pour la solidité de ses liens communautaires ${ }^{27}$ ? Répondre à cette inquiétude sociale conduirait à envisager la perception morale et la représentation anthropologique des divers âges de l'homme, de leurs embồtements naturels. Or, depuis la nuit des temps, s'affirme une «impression 
générale de pessimisme et d'hostilité à l'égard de la vieillesse», perçue comme «un mal, une infirmité, un âge triste qui prépare la mort» ${ }^{28}$. Contre ce pessimisme, au milieu du XVIII ${ }^{\mathrm{e}}$ siècle, Buffon propose la chaîne naturelle de l'existence qui lie «l'Enfance, la Puberté, l'Age viril, la Vieillesse et la Mort», évolution harmonieuse toujours vulgarisée par l'iconographie populaire dans les «degrés des âges» ou "l'Homme dans ses différents âges»" ${ }^{29}$. Vitaliste, Buffon perçoit la jeunesse comme un capital de plénitude physique que des soins attentifs peuvent «faire durer» ${ }^{30}$. Redoutée de tous, la mort s'inscrit pourtant dans la dégénérescence naturelle du corps qu'aucune volonté ne doit hâter ni arrêter. Violence inouïe à l'ordre naturel, le suicide détourne de la mort apaisée car, à l'exception des agonies provoquées par la maladie, «dans toutes les autres on meurt tranquillement, doucement et sans douleur». L'idéale «fin dernière» en ce siècle désacralisant, obsédé dès 1770 par la terreur de la «mort apparente», est donc de «mourir sans le savoir» $^{31}$.

III

«Je n'avois que trois partis à prendre, mon Cher Christin, rentrer sous la tyrannie, émigrer ou mourir. Le premier eut été une lâcheté. Le second répugne à mes principes, compromettrait mes parents et mes amis, et feroit le triomphe de mes ennemis. Je m'arrête au $3^{\circ}$ et vais rejoindre Caton. [...] S'il est quelque chose après la mort je tacherai de vous en dire des nouvelles»,

écrit ce militaire français, âgé de 34 ans, réfugié à Genève en 1793, avant de se tirer une balle dans la tête ${ }^{32}$. Eloignée de la «mélancolie» commune à maints désespérés, cette volonté du stoïcien devant les excès de la Terreur ne ressemble pas à la monomanie suicidaire définie par les aliénistes du XIX $^{\mathrm{e}}$ siècle, ni aux cauchemars de quelque Genevois, sujet de la mort volontaire, dans leurs "pensées nocturnes» ${ }^{33}$.

Entre 1650 et 1798, 299 procédures pour «suicides» concernant 300 individus témoignent de la morbidité volontaire à Genève et dans sa proche banlieue. Réprimée depuis le $\mathrm{XVI}^{\mathrm{e}}$ siècle ${ }^{34}$, la mort volontaire sera décriminalisée légalement dans la Rome protestante en 1792, fin de l'Ancien Régime genevois. Mais l'évolution pénale à l'encontre des suicidés montre une atténuation précoce des rigueurs juridiques. A l'aube du XVIII siècle, certains pasteurs de l'Eglise réformée concevaient le suicide comme une «maladie» plutôt que comme un geste du désespoir envers Dieu, alors que depuis les années 1730 une poignée de procureurs généraux évoquent la 
«mélancolie» ou l'«aliénation» pour obvier à toute criminalisation du geste fatal en convoquant ainsi les «circonstances atténuantes» classiques de la déraison ${ }^{35}$. A partir de 1738 , les biens des suicidés échappent à la saisie de l'Etat et plus aucune peine post mortem ne flétrit le cadavre d'un suicidé. Le dernier corps traîné sur la claie par les rues de la cité est celui d'une voleuse incarcérée qui s'est suicidée en 1732. Rationnelles, les autorités patriciennes de Genève étouffent volontiers la publicité d'un suicide, notamment lors des cas qui impliquent une famille attachée aux affaires de l'Etat, et ordonnent simplement des ensevelissements «sans convoi ni honneur». Procédure judiciaire stéréotypée, l'«information» sur les suicides se réduit à la levée de corps et à la constatation du corps du délit formulée par la concordance de quelques témoignages, d'une ou deux expertises de médecins pratiquant une «visite sommaire» du cadavre ou son autopsie poussée (la percée), et d'un «verbal» de l'Auditeur établissant légalement la certitude du suicide. Les chiffres montrent que les 10 suicides connus à Genève entre 1650 à 1700 représentent un taux minime de 1 pour 100000 habitants. Entre 1701 et 1750, la courbe s'élève ( 42 suicides) et atteint le taux quatre fois plus élevé de 3,8 pour 100000 habitants. Ensuite, depuis 1751 l'augmentation est forte car 248 suicides connus (population moyenne 28000 habitants) donnent un taux de 18,5 pour 100000 habitants; crue encore entre 1791 et 1798 avec le taux record de 26,4 pour 100000 habitants, comparable à ceux du XIX ${ }^{\mathrm{e}}$ siècle industriel ${ }^{36}$. Voici par la sécheresse des chiffres exprimées tout à la fois l'inquiétude des autorités formulée dès $1759^{37}$, la conjoncture séculaire genevoise de la mort volontaire et la confirmation du modèle durkheimien concernant la sur-morbidité suicidaire en pays réformé, particulièrement forte à Genève durant les crises sociales de la fin de l'Ancien Régime.

Si dans l'Angleterre puritaine de l'époque Moderne le suicide affecte surtout les couches jeunes de la population ${ }^{38}$, parmi les 300 désespérés de Genève (71\% d'hommes, $29 \%$ de femmes) émerge un groupe d'individus fauchés dans la fleur de l'âge. En effet 17 suicidés ont moins de 20 ans (5,7\% de l'échantillon), 69 sont âgés entre 21 et 30 ans ( $23 \%$ de l'échantillon) et 51 ( $17 \%$ de l'échantillon) meurent volontairement dans l'âge de la «virilité», entre 31 et 40 ans. Comme partout, les hommes de Genève se tuent trois fois plus que les femmes, alors que celles-ci sont plus jeunes que leurs compagnons lorsqu'elles se jettent au fleuve ou au lac, voire s'empoisonnent avec opium ou «eau lourde» ( $57 \%$ des femmes qui commettent un suicide se tuent avant 40 ans contre $43 \%$ chez les hommes). Forte chez les hommes et atténuée chez les femmes, la violence suicidaire concentrée en juillet et août 
est déterminée par le sexe. La noyade, mort douce, attire hommes et femmes dans une proportion proche; le poison et la «précipitation» (par une fenêtre, dans un puits) appartiennent aux désespérées, alors que la pendaison met fin à la vie de 29 hommes contre 12 femmes. Finalement, depuis $1750^{39}$, le suicide à l'arme à feu représente un privilège masculin (114 hommes et ... 1 femme), au même titre que celui commis avec une arme tranchante (4 hommes et 1 femme). Pour en terminer avec la sociologie des suicidés genevois, notons encore que les groupes sociaux privilégiés semblent plus à l'abri de la mort volontaire, bien que les magistrats et les professions libérales soient particulièrement touchés. Une sur-mortalité suicidaire affecte les individus mal intégrés dans la vie locale puisque «le nombre considérable des suicides d'étrangers (près de $25 \%$ du total)» dépasse de plus du double leur implantation démographique dans la cité ${ }^{40}$.

Si à Genève la dépénalisation du suicide est manifeste à partir du premier tiers du XVIII ${ }^{\mathrm{e}}$ siècle, et illustre à nouveau cette tolérance plus grande envers la vie privée particulière aux Etats protestants ${ }^{41}$, la mort volontaire afflige les survivants, notamment lorsqu'un individu jeune se tue au terme d'une expérience solitaire faite de révolte ou de désespoir, car le suicide juvénile est perçu comme un «raté de la communication» ${ }^{42}$ ou de la solidarité intolérable dans la sociabilité resserrée de la communauté traditionnelle. Au lendemain d'une pendaison ou d'une noyade suspecte qui ouvre le for privé des familles à l'œil du magistrat, les proches du suicidé élaborent un scénario de la normalité en évoquant les épisodes d'une vie banale censée innocenter les survivants face au geste fou de la mort volontaire.

Cette jeune cuisinière tentant en 1762 de s'empoisonner avec du mercure devient dans les témoignages de ses compagnes d'office ou de ses maîtres l'amie généreuse et pieuse, la domestique irréprochable dont on ne peut «soupçonner ni la fidélité et ni les mœurs», ni l'amitié. Son projet de mort, dissimulé jusqu'au dernier instant sous une foi religieuse ardente, était imparable ${ }^{43}$. Pourquoi a-t-elle décidé d'en finir avec la vie, cette autre jeune femme âgée de vingt-quatre ans en 1782, fille d'artisan aisé, chérie de père et sœurs, qui le soir précédant son «empoisonnement à l'opium» à dîné «joyeusement» avant de réaliser durant la veillée familiale quelque broderie entreprise... à des fins galantes? Effondré devant le cadavre de sa fille, le père tentera en vain de s'opposer à «l'ouverture juridique» du corps toujours redoutée chez les survivants, mais exigée par l'Auditeur chargé de faire confirmer par une autopsie rigoureuse le soupçon de l'empoisonnement qui détruit l'alibi de la «maladie naturelle» souvent construit par la famille ${ }^{44}$. 
Même souci de la normalité en 1783 pour cet autre père affirmant que quelques minutes avant le raptus suicidaire de son fils âgé de 23 ans

«on soupa à l'ordinaire», [puis mon fils] alla se coucher, et un moment après pendant que nous veillions encore à la cuisine, il se leva sans rien dire et se jetta par la fenêtre qui donne sur la rue (4"étage) ${ }^{45}$.

Dans un billard de Genève, les compagnons d'un jeune homme âgé de vingt ans, passionné de littérature et bientôt mort, se souviennent en 1779 d'un ami fidèle, disparu sans dette de jeu et sans «aucun motif apparent qui ait pu [le] porter à s'ôter la vie» ${ }^{46}$. Ce vigoureux grenadier âgé de vingt-quatre ans qui en 1783 s'est fait «exploser» la tête avec son fusil, abandonne des compagnons de garnison accablés se souvenant uniquement d'un ami joyeux, «sage, rangé, incapable de faire de la peine à personne» ${ }^{47}$. Si le suicide connaît quelquefois des tentatives ratées grâce à l'intervention de l'entourage ${ }^{48}$, le comportement suicidaire emprunte aussi le chemin douloureux des auto-mutilations de substitution à la mort, particulières à cet «univers de la faute ${ }^{49}$ dans lequel s'enfoncent maints désespérés de Genève. Cet adolescent âgé de 17 ans en 1761 ne se coupe-t-il pas secrètement les testicules au retour d'un vif prêche afin de se «mettre à l'abri des tentations de la chair», onze années avant de se suicider, vaincu alors par sa «seule playe [devenue] toujours plus profonde et plus terrible qui [le] porte à en venir à cette extrémité» ${ }^{50}$ ? Ici, comme toujours, la famille, notamment le père, découvre un drame que ni l'«assiduité au travail», ni le comportement «sage et régulier» ne permettaient de prévoir.

Ainsi, face aux magistrats toujours discrets au moment de l'enquête, la famille, le voisinage, les proches endeuillés, plaident pour le consensus de la normalité et évoquent le caractère «singulier» de l'issue suicidaire dont le cheminement était imprévisible. Les jeunes adultes qui se tuent sont réputés pour être d'«humeur fort douce», n'«avoir aucun goût déplacé» ou encore n'êtres affectés d'«aucune maladie, ni d'aucune tristesse». Rares sont les suicidés jeunes décrits dans les termes pathologiques de la «mélancolie» ou de la «tristesse», qui, il est vrai, apparaissent souvent comme une catégorie normative commode aux proches surpris par une mort inconcevable venant souligner les frontières fragiles entre normalité et folie. A l'exception de quelques individus malades ou atteints d'une «fièvre nerveuse» ${ }^{51}$, frappés d'un délire de persécution ${ }^{52}$, ou atteints «d'accès de mélancolie» ${ }^{53}$, les jeunes suicidés sont regardés comme «sains et normaux». La mort volontaire frappe davantage des jeunes gens à un moment d'intense activité ou de gaieté 
particulière. Cet étudiant en théologie, Lyonnais, âgé de vingt-deux ans, qui se tire un coup de pistolet au printemps 1779 , laisse un entourage atterré par une mort que l'assiduité studieuse masquait ${ }^{54}$. Ce maître horloger se souvient de la «joie de vivre» apparente de cet artisan âgé de 19 ans surpris quelques heures avant son suicide «à chanter et siffler, même [à] copier des vers» ${ }^{55}$. Ce romantisme du ruisseau est-il suffisant pour expliquer le chemin qui mène à la mort violente?

Malgré le plaidoyer pour la normalité que les proches élaborent afin de détourner le soupçon de l'abandon affectif, le suicide ombre l'«ordinaire» vie quotidienne. Circonspect, l'Auditeur s'inquiète des errances morales, des conflits familiaux, des ratés affectifs, voire des «mauvais traitements», ces «abus affreux de cette puissance que les loix n'ont déposée dans les mains des Pères que dans la persuasion qu'elles ne pouvoient le remettre en des mains plus sûres» ${ }^{56}$. Contre cet «indigne soupçon» qui ternit le sentiment familial, les proches endeuillés évoquent la solidité de la communauté qui est censée ne laisser nulle place au chagrin conduisant à la mort du désespéré. Le rappel du régime alimentaire «régulier» des jeunes suicidés, l'évocation de la «fidélité des sentiments» d'affection réciproques ou la défense de la veillée rassemblant autour du père le noyau familial, montrent combien la collectivité valorise la cohésion sociale contre la marginalisation. Amitié sans ombre, «régularité» aux cérémonies religieuses, amour de la nature ou assiduité au travail, appartiennent aussi au scénario normatif développé par les proches ou la maisonnée. En fait, par l'apologie des normes enserrant la vie des jeunes suicidés dans la sociabilité ordinaire de tous les jours, chacun affirme qu'il n'a point failli à son rôle de père, d'ami ou de maître. Déplorer le suicide de l'autre consiste finalement à rappeler combien le monde des vivants est sans reproche.

Or ce miroir du bonheur social est assombri pour les jeunes suicidés genevois, car dans leurs têtes se trouve, selon le mot inquiet d'un Auditeur, «quelquechose d'extraordinaire» qui, via le chagrin, conduit à la mort. En juillet 1753 , les prisonniers enfermés à la Discipline de Genève sont ulcérés par les pleurs puis le suicide d'un garçonnet de quatorze ans enfermé selon le vœu maternel pour «corriger» quelques turbulences domestiques ${ }^{57}$. En 1790, même appel au secours lancé par une fille de seize ans qui s'est jetée au Rhône ${ }^{58}$. Sa mère ouvrière, «séduite et abandonnée», vit d'une «manière suspecte» et semble, selon les ragots de quartier, multiplier les rencontres éphémères. A nouveau, l'adolescent suicidaire traverse un grave conflit moral accumulant abandon, «excès» de rue avec une mère qui ne «veut plus» 
son enfant et dégradation publique des rôles traditionnels familiaux. Ici, le chemin suicidaire de la jeune fille met terme à la carence affective qui attise le désespoir. Fugue solitaire, errance à travers greniers et caves des taudis populaires, ultime nuit blottie contre le corps chaud d'une servante inconnue soucieuse d'épuiser le «torrent» des larmes, et voici bientôt la jeune Ophélie du faubourg se laissant glisser à l'aube d'une journée d'été dans la turbulence mortifère du fleuve. N'a-t-elle pas confié peu avant sa mort

«qu'elle avoit une mère [mais] que c'etoit comme si elle n'en avoit pas, parce qu'elle la battoit toujours chaque fois qu'elle alloit [la] visiter»?

Ce tableau du désespoir juvénile exemplarise le chemin du suicide suivi par des individus humiliés, blessés, rejetés avec violence hors des cercles familiaux et livrés à la solitude nourricière de la mort ${ }^{59}$.

Ainsi les adolescents ou les jeunes adultes de Genève qui décident de se tuer échappent à l'image apaisante que brosse l'entourage révolté par le scandale de la mort volontaire. Motivés de diverses manières, notamment dans la dernière lettre qui devient commune depuis 1750 , les suicides concluent d'innombrables situations de détresses individuelles. Drames des familles qui se désunissent avec violence, amour malheureux, maladie du corps ou «douleur de l'âme», sensibilité extrême à la différence individuelle, réparation outrée d'un honneur «défait», catastrophe économique, ou encore sentiment de religiosité exacerbé, les chemins du suicide étaient innombrables et seul Dieu devait en connaître les méandres. Malgré l'hétérogénéité des comportements suicidaires, la mort volontaire des jeunes adultes montre les points fragiles de l'ancrage social dans la communauté traditionnelle protestante, qui ne connaît nulle autorité morale susceptible de purger les chagrins de l'âme ou de dénouer avec neutralité les conflits intimes. Brisant la norme communautaire, le suicide juvénile exprime une réponse solitaire à des agressions extérieures devenues insupportables car peu exprimables à l'extérieur du foyer familial, qui est souvent à la source du chemin suicidaire. Contre la réprimande violente administrée «selon le devoir d'un maître et d'un Pasteur», une jeune femme avale du poison; «séduite» et engrossée une autre servante âgée de vingt-cinq ans tente aussi de s'empoisonner ${ }^{60}$. Contre le rejet familial et la violence de géniteurs vite devenus «indignes» sous la plume des magistrats, un garçonnet se pend, deux frères se jettent par une fenêtre, une adolescente se noie, un jeune homme plonge dans l'écume blanche qui libère. Contre le veto paternel devant l'engagement amoureux réciproque, une jeune femme s'empoisonne 
et son amant se tire une balle après avoir embrassé le «cadavre tiède de sa maîtresse» déjà livide. A la suite d'emprisonnements, toujours perçus infamants durant l'Ancien Régime, de jeunes hommes retiennent la corde plutôt que le déshonneur d'un procès ${ }^{61}$ puisque, convient ce magistrat de Genève,

«dans l'ordre des biens, la vie n'est pas toujours le premier, l'honneur et la réputation le précédent, et quiconque prefereroit à ne pas exister honteusement dans l'opinion des autres, seroit tout à la fois un lâche et un insensé» ${ }^{62}$.

Contre une faillite brisant l'honneur, ou contre les excès politiques d'un monde qui semble être devenu fou, des hommes pleins de force choisissent l'impact d'une balle mortelle plutôt que de continuer à vivre le «cœur empoisonné par les chagrins».

En effet, quel «plaisir peut prendre quelqu'un par exemple qui dès sa plus tendre jeunesse est obligé de plier sans cesse sous le joug d'une volonté arbitraire et capricieuse qui étouffant en lui ses inclinations naturelles prépare de loin sa ruine et lui creuse un abîme dont il ne pourra se tirer que par des causes tout à fait extraordinaires» ${ }^{63}$ ?

Expression d'un individualisme exacerbé butant sur les contraintes imposées par la collectivité, le suicide juvénile, commis surtout à la belle saison de l'année, apporte par la douleur une liberté qui effraie les survivants puisque la mort volontaire altère la représentation sociale de la normalité familiale ou les engagements de l'amitié.

Le suicide «tragédie individuelle» ou «maladie sociale»? Le dilemme kantien du «rebelle social» ${ }^{64}$ ne se discute pas encore dans les termes du psychanaliste ou du déterminisme sociologique au cours du XVIII ${ }^{\mathrm{e}}$ siècle, bien que le suicide soit souvent perçu comme un signe d'anormalité individuelle, semblable peut-être aux déviances sexuelles déférées devant les tribunaux criminels. Mais à l'opposé des suicides commis par des adultes plus âgés, voire des vieillards, à la suite d'un retour de fortune ${ }^{65}$, d'une maladie douloureuse ou d'un deuil insurmontable, la mort volontaire qui frappe les rangs de la jeunesse de Genève dans une proportion sensible au fil du siècle horrifie la communauté. A chaque fois, en effet, les survivants sont ulcérés par le suicide d'un individu jeune puisque, avant de «quitter la vie», le désespéré «abandonne» famille et amis livrés au cortège sombre des remords, de la solitude et du chagrin. Avant la «mort de toi», c'est bien le «chagrin de moi» qui affecte le cercle des proches endeuillés par un suicide juvénile. D'autre part, soulignée par magistrats ou survivants confrontés aux têtes fracassées ou aux corps rongés par la vase putréfactrice, l'atteinte mortelle «à la belle physionomie» et à la santé du corps scandalise. Drame individuel, 
le suicide juvénile est certainement perçu comme l'aggravation de la «vie fragile», voire comme une menace supplémentaire sur un régime démographique réputé en reflux ${ }^{66}$. La mort volontaire, «contraire à la nature» à écouter magistrats et certains suicidés eux-mêmes qui abandonnent une dernière lettre, voire des réflexions sur le suicide ${ }^{67}$, met un terme arbitraire, tout comme la maladie soudaine, à une existence éloignée de sa maturité. Le scandale provoqué par le suicide juvénile souligne en fait l'existence d'une double jeunesse. D'un côté, une jeunesse pleine de vitalité, responsable des aînés, et devant assurer les lendemains du genre humain mais qui est fauchée par une mort volontaire que l'entière communauté condamne. De l'autre, une jeunesse morale qui provoque la «compassion» car la mort volontaire semble naître d'une «fragilité de l'âme» toujours évoquée par les survivants au moment de s'interroger sur le geste fatidique d'un jeune adulte. Est-ce au carrefour de cette double représentation du printemps de la vie que le suicide juvénile souligne la socialisation radicale de la jeunesse dans la communauté traditionnelle organisée autour des aînés? Fauchés dans la jeunesse de la vie, les suicidés de Genève sont-ils alors accablés par cet «ennui de vivre [qui] l'emporte sur l'horreur de mourir» souligné par la correspondance imaginaire d'une fiction célèbre ${ }^{68}$ ? Werther, ultime interlocuteur de quelques jeunes suicidés, n'explique certainement pas le suicide juvénile, mais évoque l'intériorisation d'une culture nouvelle qui autorise à donner une forme stéréotypée à la mort volontaire traditionnelle. Le texte du poète, taché de sang et de cervelle dans l'alcôve de la mort, prolongerait le message de la dernière lettre toujours pétrie de foi chrétienne, miroir affiné des chagrins personnels laissés en souvenir aux vivants endeuillés par la mort de la belle jeunesse. 


\section{Notes}

1 Les sources judiciaires, procès criminels série $\mathrm{I}$ [depuis maintenant $\mathrm{PC}$ ], proviennent tous des Archives d'Etat de Genève.

2 Cet article s'inscrit dans une recherche globale sur la dimension sociale de la justice criminelle genevoise durant le XVIII' siècle: M. Porret, «Mourir sur l'échafaud à Genève au XVIII' siècle», Déviance et société, décembre 1991, XV, 4, pp.381-405. Idem, "Viols, attentats aux mœurs et indécences: les enjeux de la médecine légale à Genève (16501815)», à paraître, Equinoxes, novembre 1992, (Histoire et histoire de la médecine). Idem, Le crime et ses «circonstances». Punir à Genève au XVIII e siècle : institutions, discours, pratiques. Université de Genève, thèse de doctorat dactylographiée, 774 p., à paraître.

3 R. Roth, «Réformes du droit pénal à Genève durant l'époque révolutionnaire», in : Regards sur la révolution genevoise 1792-1798, [volume publié] par L. Binz, B. Baczko, M. Neuenschwander, O. Labarthe, R. Durand, Genève 1992, pp.151-167.

4 A. Bayet, Le suicide et la morale, Paris 1922, [reprint] Arno Press, NY, 1975, p. 634.

5 Réitération dans les ordonnances royales des dispositions pénales infamantes à l'encontre du suicide en 1712, 1736, 1770; une atténuation survient pourtant en 1770 avec l'abandon de la flétrissure corporelle du corps du suicidé... dont seule la mémoire est «jugée». Sur le Code pénal, P. Lascoumes, P. Poncela, P. Lenoël, Au nom de l'ordre. Une histoire politique du code pénal, Paris, 1989.

6 A l'exception du suicide antique, on retiendra ici: J.-C.Schmitt, «Le suicide au Moyen Age», Annales E.S. C., 1976, I, pp. 3-28: M. Mac Donald, «The Secularization of suicide in England 1660-1800», Past and Present, 111, 1986, pp.50-97; T. R. Murphy, «Woful Childe of Parents Rage»: Suicide of Children and Adolescents in Early Modern England 15071710», in: The Sixteenth Century Journal XVII, 1986, pp. 259-270. L. Haeberli, «Le suicide à Genève au XVIII 'e siècle», in: Pour une Histoire Qualitative, Etudes offertes à Sven StellingMichaud, Genève 1975, pp. 115-129. O. Anderson, «Did Suicide increase with Industrialization in Victorian England?», in: Past and Present, 86, 1980, pp. 149-173, et Suicide in Victorian and Edwardian England, Oxford 1987. B. T. Gates, Victorian suicide. Mad Crimes and Sad Histories, Princeton, New Jersey, 1988. L. V.Thomas, Anthropologie de la mort, Paris 1975, notamment pp. 169-175.

7 R. Favre, La Mort au Siècle des Lumières, Lyon 1978, p. 470; en Angleterre le mot se répand autour de 1630, M. Mac Donald, op. cité (voir n. 6), p. 53.

8 S. A. Tissot, «De l'épilepsie», 1770, in: Des Nerfs et de leurs maladies, Euvres complètes, Paris 1813, X, p. 387.

9 A. Bayet, op. cité (voir n.4), p. 8.

10 Abbé F. de la Mennais, «Sur le suicide», Le Conservateur V, 54, 1819, pp. 57-62.

11 E. Esquirol, Des maladies mentales considérées sous les rapports médical, hygiénique et médico-légal, 2 vols., Paris 1838, «Du suicide», I, pp.259-331; A. Brierre de Boismont, Du suicide et de la folie suicide, Paris 1865, particulièrement les pages 212, 216, 461 et 623 sur la folie-suicide.

12 Esquirol, op. cité, p. 286.

13 E.Durkheim, Le Suicide. Etude de sociologie, Paris 1897, [réédition], Paris 1960; M. Halbwachs, Les causes du suicide, Paris 1930, [reprint] Arno Press, New York 1975.

14 PC 13346. Présence avérée de Werther auprès de jeunes suicidés: PC 14170, 1783 PC 17092, 1792 ; présence vraisemblable, PC 13889, 1782. 
15 M. Porret, «L'adieu des suicidés (1750-1790)», communication inédite, colloque international «savoir mourir», Université Paris XII (UFT «Communication et insertion dans la société, département Santé et société), 21-23 mai 1992.

16 Brierre de Boismont, op. cité (voir n.11), p.272 et aussi pp.296-331; R. Cobb, Death in Paris, Oxford 1978, p.118-119.

17 Allemagne: H. Brunschwig, La crise de l'Etat prussien à la fin du XVIII siècle et la genèse de la mentalité romantique, Paris 1947, pp.267-268. France: R. Favre, op.cité (voir n.7), pp.413-466; M. Vovelle, La mort et l'Occident de 1300 à nos jours, Paris 1983, pp.476-478. Angleterre: M. Mac Donald, op. cité (voir n. 6), p. 83.

18 S. Mercier, Tableau de Paris, 1782, III, chap. 258. R. Cobb, op. cité.

19 Jeunesse et suicide: A. Brierre de Boismont, op.cité (voir n.11), pp.599-608, 630-632. L. V. Thomas, op. cité (voir n. 6), pp. 302-310. J. Baechler, Les suicides, Paris 1975, pp. 389$393,476-479$.

20 Cité d'après Bayet, op. cité (voir n.4), pp. 650-651.

21 J. B. C. Delisle de Sales, La Philosophie de la Nature, 3 vols., [édition de] Paris, 1770, III, pp. 217-264, III, p. 45.

22 Ibidem, III, pp. 126, 148, 155.

23 [Chevalier de Jaucourt], «Jeunesse» (Oecono[mie] anim[ale]), Encyclopédie.

24 Pour Tissot les passions extrêmes (amour, jeu, etc.) engendrent la maladie, Des nerfs et de leurs maladies, op. cité (voir n. 8), IX, pp. 235-239.

25 S. A. Tissot, L'Onanisme, dissertation sur les maladies produites par la masturbation, 1760 in: op. cité, III, pp. 216, 217, 230 et 290. Esquirol, op.cité (voir n. 11), I, p. 288.

26 E. Esquirol, op. cité, p. 289.

27 P.Laslett, The World We Have Lost. England Before the Industrial Age, New York 1965, suicide et communauté traditionnelle, pp.136-138.

28 G. Minois, Histoire de la vieillesse en Occident, de l'Antiquité à la Renaissance, Paris 1987, p. 407 .

29 N. Garnier, L'imagerie populaire française, Paris 1990, notices 849 et 912.

30 G. de Buffon, «De la vieillesse et de la mort», cité d'après J.L. Binet et J. Roger, Un autre Buffon, [anthologie], Paris 1977, pp.96-116.

31 Ibidem, pp. 107-108.

32 PC 17079, 1793.

33 M. Porret, «Les «Pensées nocturnes〉 de Pierre Frémont, libraire et «explicateur de songes` à Genève au XVIII' siècle», La Nouvelle Revue Française, 449, 1990, pp.108-133; ibidem, «Rêver de s'enrichir ou s'enrichir en rêvant. Les 〈pensées nocturnes` du Genevois Pierre Frémont, «dit Butini〉, libraire et explicateur de songes (1774)», Revue d'histoire moderne et contemporaine I, 1991, pp. 22-50.

34 L. Haeberli, op.cité (voir n.6), p. 118, une procédure de 1542 atteste du premier cas de suicide juridiquement connu à Genève et qui ouvre une petite série de 30 dossiers conservés jusqu'en 1650.

35 PC 4679, 1686; PC 8043, 1733; PC 8277, 1735.

36 L. Haeberli, op.cité (voir n.6), pp.117-118. Sur la statistique de l'anomie suicidaire à Genève au XIX' siècle, J.-M. Dufour, Etude statistique et comparée sur le suicide à la morgue de Genève (1800-1900), Marseille 1903.

37 Archives d'Etat de Genève, Registre du Conseil (RC) 1759, «Comme il a été interjetté que le 
nombre de ceux qui se donnent volontairement la mort augmente depuis plusieurs années, et qu'il serait à désirer d'aviser d'arrêter cette fureur, Mons[ieur] le Premier [syndic] a dit qu'il mettroit la matiere sur le tapis», fol. 57.

38 T. R. Murphy, op.cité (voir n. 6), sur 1098 suicidés dont l'âge est connu, 416 individus (2 suicidés sur 5) sont âgés de 21 ans ou moins, dont 192 qui se tuent avant leur quinzième année. Globalement entre 1485 et 1714, 67\% des suicidés meurent entre 11 et 40 ans (âge moyen 30 ans); R. Cobb, op.cité (voir n. 16), la jeunesse des noyés parisiens à la fin du XVIII siècle est plus atténuée, car $51 \%$ sont âgés de moins de 39 ans, p. 112 .

39 Jusqu'en 1750 les hommes se noient aussi volontiers que les femmes et les coups de feu sont rares dans les greniers du désespoir; voir M. Mac Donald, op.cité (voir n.6), p.66, et R. Cobb, op. cité (voir n. 16), pp. 25-28, et sur le suicide masculin commis avec une arme à feu, ibidem, pp. 127-129.

40 L. Haeberli, op. citê (voir n. 6), p. 119.

41 La décriminalisation du suicide dans la Hollande protestante survient précocement dès 1658, P.Spierenburg, The Spectale of Suffering. Executions and the evolution of repression: from a preindustrial metropolis to the European experience, Cambridge [...], 1984, pp. 56-57; pour l'Angleterre, M. Mac Donald, op. cité (voir n. 6), pp. 93-96.

42 L. V. Thomas, op. cité (voir n. 6), pp. 307-308.

43 PC 10999, avril 1762.

44 PC 13889, mai 1782. Même scénario familial, PC 13369, août 1779; PC 14629, mai 1785 : tentative de camouflage du suicide en une mort accidentelle. PC 14098, 1786, hostilité familiale semblable face à l'autopsie confirmant le suicide.

45 PC 14131, juillet 1783.

$46 \quad$ PC 13346, juin 1779.

47 PC 14137, août 1783.

48 PC 10999, avril 1762, tentative d'empoisonnement d'une jeune servante âgée de 20 ans, congédiée et humiliée par les allégations de son maître, pasteur et professeur, au sujet de son honnêteté; PC 11218; février 1764, tentative d'auto-égorgement d'un jeune homme de 27 ans frappé de crises de «mélancolie» qui s'ouvre la gorge avec un rasoir dont la lame mesure 12 centimètres (pièce à conviction).

49 Y. Pelicier, «Le suicide, milieux et modèles». Revue de Psychologie des Peuples, I, 1967, pp. 47-72.

50 PC 10868, 1761, «mutilation», et PC 12363 , octobre 1772, dernière lettre, suicide commis avec un fusil de gros calibre.

51 PC 14131, juillet 1783, cité; PC 14 091, juin 1783, noyade volontaire d'une jeune femme affectée de «maux de tête» atroces.

52 PC 10091, juin 1754, pendaison après une tentative de noyade qui échoue d'un homme qui crie publiquement que «les Savoyards devoient le prendre et le faire mourir».

53 PC 12450 , mai 1773, cité; PC 11 218, février 1764, «tentative de suicide»; selon son propre témoignage, le rescapé était «sujet à des mouvemens convulsifs dans les nerfs qui lui attaquoient le cerveau et le mettoient hors des sens, hier environ les cinq heures du soir, il luy en prit un acces si violent, qu'il se porta à se couper la gorge avec un rasoir. Qu'etant revenu à lui-même il en demande ardemment pardon à Dieu». De tels symptômes ressemblent à la nosographie de l'épilepsie proposée par Tissot, «Des nerfs et de leurs maladies», chapitre X, «De l'épilepsie», Euvres complètes, Paris 1813, X, pp. 2-23. 
$54 \quad$ PC 13322 , avril 1779.

55 PC 14170, septembre 1783, cité.

56 PC 11373, 1765, «excès sur ses enfants» [défenestration], Conclusions du Procureur général Jean-Robert Tronchin.

57 PC 9989, juillet 1753.

58 PC 16195, 1790.

59 La rupture avec la famille est au centre de maintes procédures pour suicides; par exemple, PC 8080 , juillet 1737 , noyade «toute habillée», après deux semaines de fugue, d'une jeune femme de 20 ans, orpheline ou fille de divorcés, pourtant «recherchée en mariage», mais brisée par une terrible dispute avec sa tante chez qui elle vit et qui la menace de «bannissement» (témoignage de l'oncle); PC 14163, septembre 1783, noyade volontaire devant une foule de curieux d'un homme âgé de 20-25 ans affecté de «lourds chagrins» familiaux et qui «avoit des désagréméns avec sa mère qui etoit fort vive» (témoignages confondus des proches), etc. Le suicide juvénile et les conflits familiaux sont soulignés par T. R. Murphy, op.cité (voir n.6), pp. 260 et 269, et d'une manière atténuée par R.Cobb, op. cité (voir n. 16), pp. 107-108. Sur les violences familiales à Genève, M. Porret, «Entre tolérance tacite et intolérance collective: enfants violentés et parents dénaturés, Genève 1700-1765», Revue du Vieux Genève 1989, pp. 27-43.

60 PC 11312, novembre 1764.

61 PC 13544, août 1779, suicide en prison d'un tailleur âgé de 23 ans. PC 14 744, octobre 1787, pendaison dans une cellule de la «Discipline» d'un jardinier âgé de 26 ans, arrêté pour avoir dérobé 335 florins à son maître.

62 PC 11219, 1764, «duel», jurisconsulte éclairé en matière pénale Jean-Robert Tronchin, Procureur général de 1760 à 1767 , est resté célèbre pour avoir requis contre l'Emile et le Contrat Social en 1762.

63 PC 11 902, juillet 1769, «Réflexions sur le suicide», 14 feuillets, manuscrit inédit que nous sommes en train de publier.

64 M. J. Seidler, «Kant and the Stoics on Suicide», Journal of the History of Ideas XLIV, 1983, pp. 429-453.

65 Entre 1740 et 1790, de nombreux suicides sont commis par des femmes et des hommes «assistés» par les diverses «Bourses» de Genève et quelquefois «détenus» à l'Hôpital général. Le diagnostic établi par l'entourage valorise volontiers alors la «mélancolie».

66 S.A.Tissot, Avis au peuple sur sa santé, 1761, Op.cité, I, «Introduction», thème de la dépopulation surtout pp.41-51.

67 PC 11902, cité.

68 J. J. Rousseau, La Nouvelle Hélö̈se, III, lettre XXI, citation selon l'édition donnée par Michel Launay, Garnier Flammarion p. 281. 


\section{Summary}

\section{Juvenile suicide at Geneva in the $18^{\text {th }}$ century}

Whether it is the unhappy ending of family grief, the only solution to an unrequited love or due to some pathological behaviour, juvenile suicide horrifies the community of the Ancien Régime, which tends to interpret it as an "illness of the soul" characteristic of a youth that combines bodily vitality with moral frailty, but whose role would be nonethless to ensure the morrows of mankind. Juvenile suicide underlines the flaws of social solidarity in the tightly knit community of the Ancien Régime, and it deepens the social resentment against wilful death which, since 1774 , has often been attributed to the harmful influence of the kind of "philosophy" or free will advocated by Goethe's unfortunate Werther.

\section{Zusammenfassung}

\section{Der jugendliche Selbstmord in Genf im 18. Jahrhundert}

Ob es der unglückliche Schluss eines Familienkummers ist, die einzige Lösung einer traurigen Liebesgeschichte oder das Zeichen eines pathologischen Verhaltens, jugendlicher Selbstmord entsetzt die Gemeinschaft des Ancien Régime, die ihn verstehen will als eine «Krankheit der Seele», bezeichnend für eine Jugend, die körperliche Vitalität mit seelischer Schwäche verbindet, die aber für die Zukunft der Menschheit sorgen müsste. Jugendlicher Selbstmord hebt die Schattenseite der sozialen Solidarität der eng verbundenen Gemeinschaft des Ancien Régime hervor, und er verstärkt den gemeinschaftlichen Groll gegen den freiwilligen Tod, der seit 1774 oft dem schädlichen Beispiel der «Philosophie» oder des freien Willens des armen Werther zugeschrieben wurde.

Michel Porret, Dr ès lettres

Université de Genève

Département d'histoire générale

1205 Genève 